\title{
Diagnostic immunohistochemistry in gynaecological neoplasia: a brief survey of the most common scenarios
}

\author{
Elisabetta Kuhn, ${ }^{1,2,3}$ Ayse Ayhan ${ }^{4,5}$
}

\begin{abstract}
${ }^{1}$ Pathology Unit, Arcispedale Santa Maria Nuova-IRCCS, Reggio Emilia, Italy ${ }^{2}$ Department of Morphology, Surgery and Experimental Medicine, University of Ferrara, Ferrara, Italy

${ }^{3}$ Laboratory of Technology for Advanced Therapies (LTTA), University of Ferrara, Ferrara, Italy

${ }^{4}$ Departments of Pathology, Hamamatsu and Hiroshima Universities Schools of Medicine, Seirei Mikatahara Hospital, Hamamatsu, Japan

${ }^{5}$ Department of Pathology, Johns Hopkins University School of Medicine, Baltimore, Maryland, USA
\end{abstract}

\section{Correspondence to} Dr Elisabetta Kuhn, Department of Morphology, Surgery and Experimental Medicine and Laboratory of Technology for Advanced Therapies Centre, University of Ferrara, Ferrara 44121, Italy; elisabettakuhn@ hotmail.it and Professor Ayse Ayhan, Department of Pathology, Hamamatsu University School of Medicine, Handayama 1-20-1, 4313192 Hamamatsu, Japan; ayseayhanjp@gmail.com

EK and AA contributed equally.

Received 12 September 2017 Revised 2 November 2017 Accepted 3 November 2017 Published Online First

7 December 2017

\begin{abstract}
Immunohistochemistry is a valuable adjunct in routine gynaecological pathology. The molecular revolution has redesigned knowledge of gynaecological cancers and refined histological classification. The direct consequence has been the progressive introduction of new immunostainings for diagnostic and classification purposes. Hence, we review the routine diagnostic use of immunohistochemistry in the field of gynaecological neoplasia. We reviewed the immunomarkers useful in gynaecological pathology according to literature revision, our personal experience and research findings. We discuss the application of immunohistochemistry to reach the most accurate diagnosis in morphologically equivocal cases of gynaecological pathology and present the appropriate panel of immunomarkers in the most common scenarios of gynaecological pathology. This short review provides an updated overview of the essential immunohistochemical markers currently used in the diagnostics of gynaecological malignancies along with their molecular rationale.
\end{abstract}

\section{INTRODUCTION}

Immunohistochemistry (IHC) combines microscopic morphology with accurate molecular identification and allows in situ visualisation of any specific protein antigen. The introduction of IHC in diagnostic pathology has revolutionised routine practice, and IHC studies have significantly contributed to a better understanding and subtyping of many malignancies, initially lymphoid neoplasms. Furthermore, IHC has become an integral part of the definition of the majority of solid tumours and is progressively gaining a foothold in guiding anticancer therapy. Among other examples, HER2/neu and oestrogen receptor (ER) expression is routinely used to identify patients with breast cancer eligible to trastuzumab and tamoxifen, respectively.

With the boost and consequential widespread use of advanced technologies, molecular studies that claim to have discovered novel candidate makers with diagnostic, predictive, prognostic or therapeutic value are published daily. In this context, the responsibility of the pathologist is increasing. Besides making tissue diagnosis, they are also in charge of (1) guaranteeing the adequacy of samples used for diagnostic tests, which will be translated into therapeutic decisions, (2) performing IHC biomarker analysis and (3) assisting the development of novel tissue biomarkers. Over the last decade, molecular studies have unveiled the molecular genetic pathway of gynaecological malignancies and enriched the portfolio of IHC markers useful in the differential diagnosis of gynaecological diseases. Accordingly, IHC represents a solid adjunct for the classification of gynaecological malignancies that improves interobserver reproducibility ${ }^{1}$ and has the potential of revealing unexpected features. However, interpretation in the light of knowledge-based specificity of each single marker along with histopathology expertise and stringency is still the sine qua non. A satisfactory IHC must localise cells and tissue targets, clearly and specifically, keeping the non-specific background to a minimum level.

Here, we will describe the panels of IHC markers used in the most common scenarios of differential diagnosis seen in routine gynaecological pathology, along with their rationale. Though beyond the scope of this paper, clinical information and macroscopical and microscopical features will be outlined at times since they still represent a keystone for the correct diagnosis and characterisation of many pathological entities.

\section{LOOKING AT THE OVARY}

Primary ovarian tumours are summarised in three main subgroups with well-defined clinicopathological characteristics: epithelial, germ-cell and sex-cord stromal tumours (table 1). ${ }^{2}$ However, metastatic tumours and primary tumours derived from non-ovarian-specific lymphoid or stromal cells (ie, lymphomas, leukaemias and soft-tissue tumours) should not be ignored since they represent a large proportion of ovarian malignancies.

Currently, PAX8 is emerging as the most specific marker to distinguish a primary ovarian carcinoma from a metastasis, but it lacks sensibility as it is also expressed in metastasis from the endocervix, kidney and thyroid (see figure 1 and table 2). ${ }^{1}$ The most common malignancy of the ovary is highgrade serous carcinoma (HGSC) that together with other serous tumours of the adnexa (low-grade serous carcinoma and borderline serous tumour) are now thought to derive from the fallopian tube epithelium. ${ }^{3}$ Coherently, these tumours demonstrate consistent nuclear expression of Müllerian marker WT1, which is highly expressed in the normal tubal epithelium. ${ }^{3}$ WT1 is the most sensitive and specific marker for serous histotype and can be used to discriminate serous tumours from all other histotypes.

Recently, a practical approach to the use of IHC in the classification of primary ovarian carcinomas 


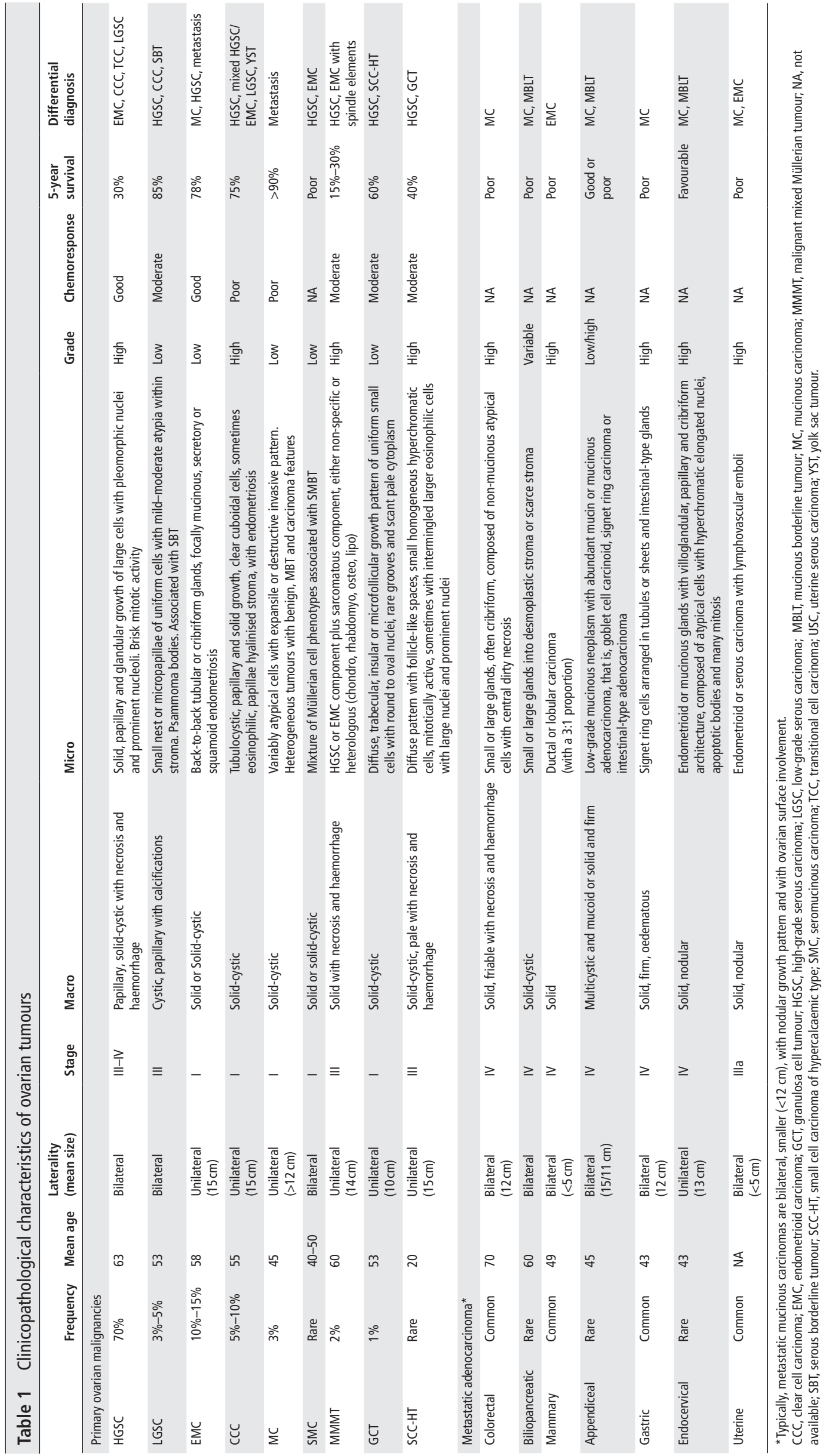




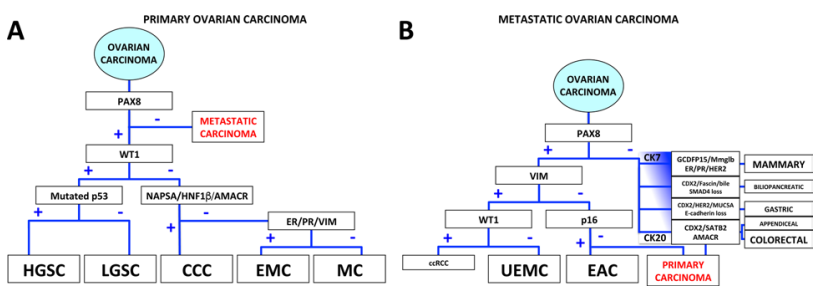

Figure 1 Immunohistochemical algorithm proposed to diagnose ovarian carcinomas, both primary and metastatic. (A) This algorithm addresses the distinction of morphologically equivocal primary ovarian carcinomas using five to nine immunomarkers. (B) The stepwise immunohistochemical approach for metastatic ovarian carcinomas uses six main immunostainings plus other tissue-specific markers. Because CK7 and CK20 are often coexpressed, they are schematically represented as a continuous vertical from prevalent CK7 positivity (upper end) to prevalent CK20 positivity (lower end). The frequency of the metastatic disease is correlated with font size. CCC, clear cell carcinoma; ccRCC, clear cell renal cell carcinoma; CK, cytokeratin; EAC, endocervical adenocarcinoma; EMC, endometrioid carcinoma; ER, oestrogen receptor; GCDFP15, gross cystic disease fluid protein-15; HGSC, high-grade serous carcinoma; LGSC, low-grade serous carcinoma; MC, mucinous carcinoma; Mmglb, mammaglobin; NAPSA, Napsin A; PR, progesterone receptor; UEMC, uterine endometrioid carcinoma; VIM, vimentin.

has been proposed and presented as an IHC algorithm. ${ }^{1}$ This algorithm results in a hierarchical decision tree, and takes advantage of four IHC markers, including WT1, p53, Napsin A and progesterone receptors (PR), to subclassify primary ovarian carcinomas. We propose a modified IHC algorithm (figure 1A) that includes other interchangeable markers, namely HNF1 $\beta$ and AMACR (also known as racemase or p504S) for clear cell carcinoma (CCC), and ER and vimentin for endometrioid carcinoma, to gain flexibility in routine practice. Moreover, we propose an algorithm (figure 1B) for approaching either ovarian carcinomas with an unusual morphology, with regard to ovarian primary, or suspected metastasis of unknown origin, that keeps into account both the frequency of metastasis and the morphological similarity.

Endometrioid carcinoma versus high-grade serous carcinoma

HGSC with glandular and cribriform growth may closely resemble endometrioid carcinoma. In addition to WT1 expression, virtually all HGSCs show an aberrant p53 protein expression due to TP53 somatic mutation. ${ }^{4}$ Specifically, in HGSC p53 is overexpressed, in that it is diffusely and intensely nuclear positive, in $60 \%-70 \%$ of HGSCs because of missense mutation, whereas it is entirely negative in the remaining cases due to truncating mutation. Notably, p53 may also be aberrant in almost 30\% of endometrioid carcinomas, particularly in those with high nuclear grade. Besides this, p16 expression tends to be intense and diffuse or completely blank in HGSC, while in endometrioid carcinoma, it shows a 'mosaic' pattern. Usually, endometrial carcinoma expresses cytoplasmic vimentin, as opposed to HGSC. Half of endometrioid carcinomas harbour CTNNB1 mutations, which cause nuclear translocation of $\boldsymbol{\beta}$-catenin, differently from HGSC. ${ }^{5}$ More recently, ARID1A somatic mutation and concurrent protein expression loss have been identified in up to $50 \%$ of endometrioid carcinomas. ${ }^{7}$ Also, the tumour suppressor PTEN is mutated and the protein lost in almost $70 \%$ of endometrioid carcinomas; IHC downregulation for PTEN is also reported in up to $52 \%$ of HGSCs due to either homozygous

Table 2 Immunohistochemical (IHC) markers in the differential diagnosis of ovarian tumours

\begin{tabular}{|c|c|c|c|c|c|c|c|c|c|c|c|}
\hline & PAX8* & WT1† & ER & p53 & ARID1A & $\beta$-cat & calr & CK7 & CK20 & CEA & Specific \\
\hline \multicolumn{12}{|c|}{ Primary ovarian tumours } \\
\hline HGSC & + & + & + & $\mathrm{M}$ & wt & wt & & + & - & - & p16 \\
\hline LGSC & + & + & + & wt & wt & wt & $-1+$ & + & - & - & KRAS mut, BRAF mut \\
\hline EMC & + & - & + & $w t>M$ & $M>w t$ & $M>w t$ & - & + & - & - & $\begin{array}{l}\text { PTEN and MMR loss } \\
\text { Presence of endometriosis }\end{array}$ \\
\hline $\mathrm{CCC}$ & + & - & $-1+$ & wt & $M>w t$ & wt & & + & - & - & Napsin A, HNF1 $\beta$, AMACR \\
\hline $\mathrm{MC}$ & + & - & - & $w t>M$ & wt & wt & - & + & + & $-1+$ & $\begin{array}{l}\text { Presence of teratoma or Brenner } \\
\text { tumour }\end{array}$ \\
\hline SMT & + & - & + & wt & $M>w t$ & wt & - & + & - & - & Presence of endometriosis \\
\hline GCT/SCST & $-1+$ & + & $+l-$ & wt & wt & wt & + & - & - & - & $\begin{array}{l}\text { Inhibin, SF1, FOXL2 mut (GCT), } \\
\text { DICER1 mut (SCST), EMA }\end{array}$ \\
\hline SCC-HT & + & + & - & $\mathrm{M}$ & wt & wt & + & - & - & - & SMARCA4 (BRG1) loss \\
\hline \multicolumn{12}{|c|}{ Metastatic adenocarcinomas } \\
\hline Colorectal & - & - & - & $w t>M$ & wt & $\mathrm{M}>\mathrm{wt}$ & - & - & + & + & CDX2, SATB2 \\
\hline Biliopancreatic & - & - & - & $M>w t$ & wt & wt & - & + & + & + & SMAD4 (DPC4) loss \\
\hline Breast & - & - & $+1-$ & $w t>M$ & wt & wt & - & + & - & - & GCDFP15, mammaglobin \\
\hline Lung & - & - & - & $w t>M$ & wt & wt & - & + & - & - & TTF1, Napsin A \\
\hline Kidney & + & + & - & wt & wt & wt & - & $-1+$ & - & - & CD10, vimentin, HIF1 $\beta$ \\
\hline Endocervix & + & - & $-1+$ & $w t>M$ & wt & wt & - & + & - & + & p16, HPV \\
\hline Uterine EMC & + & - & + & $w t>M$ & $M>w t$ & $M>W t$ & - & + & - & - & Vimentin, PTEN and MMR loss \\
\hline USC & + & $-1+$ & $-1+$ & $M$ & wt & wt & - & + & - & - & Vimentin \\
\hline
\end{tabular}

ARID1A M consists in the loss of IHC staining, while ARID1A wt is nuclear staining. $\beta$-cat $\mathrm{M}$ is nuclear positivity; $\beta$-cat wt is cytoplasm, membrane positivity or negativity. $\mathrm{p} 53 \mathrm{M}$ corresponds to intense and diffuse positivity in $\geq 60 \%$ of cells or complete negativity; p53 wt is the presence of rare cells weakly positive or positivity in $<60 \%$ of cells.

${ }^{*}$ PAX8 is also expressed in kidney, thyroid, parathyroid and thymic carcinomas.

tWT1 stains also normal mesothelium, kidney, their derived neoplasias and desmoplastic small round cell tumour.

$\beta$-cat, $\beta$-catenin; calr, calretinin; CCC, clear cell carcinoma; CK, cytokeratin; EMC, endometrioid carcinoma; GCT/SCST, granulosa cell tumour/sex-cord stromal tumour; HGSC, highgrade serous carcinoma; LGSC, low-grade serous carcinoma; M, mutant pattern; MC, mucinous carcinoma; MMR, DNA mismatch repair genes; mut, mutation; SCC-HT, small cell carcinoma of hypercalcaemic type; SMT, seromucinous tumour; USC, uterine serous carcinoma; wt, wild-type pattern. 
deletion or hemizygous loss. ${ }^{8}$ Moreover, cyclin E1 expression and CCNE1 amplification have been reported in many HGSCs, but are absent in endometrioid carcinomas. ${ }^{9-11}$

\section{Clear cell carcinoma versus high-grade serous carcinoma}

Distinguishing HGSC with cytoplasmic clearing from CCC, or vice versa CCC with eosinophilic cytoplasm from HGSC, may be challenging. Useful markers include WT1 and ER chiefly expressed in HGSC, along with aberrant p53, as well as nuclear HNF1 $\beta$ and cytoplasmic Napsin A and AMACR positive in CCC, ${ }^{12}$ and negative ARID1A in up to $57 \%$ of CCCs.

\section{Advanced ovarian versus uterine serous carcinoma}

Disseminated serous carcinomas that contemporarily involve ovaries, uterus and peritoneum represent a challenge for delineating the site of origin. WT1 is the most reliable marker in this setting and marks HGSC diffusely and uterine serous carcinoma (USC) only in up to one-third of cases, in a variable way. Specifically, in cases of synchronous involvement of both the endometrium and ovaries, WT1 is mainly worthy if it is negative at both locations, supporting an endometrial primary, or if the staining patterns are different at the two sites, suggesting two independent tumours. ${ }^{13-15}$

Peritoneal serous carcinoma versus epithelioid mesothelioma Epithelioid mesothelioma may closely resemble serous carcinoma, both low-grade and high-grade. Among the useful immunomarkers to distinguish these neoplasias, there are calretinin, keratin 5/6 and D2-40 positive in mesothelioma and PAX8, ER, claudin4, MOC31 and Ber-EP4 expressed by serous carcinoma. ${ }^{16} 17$ Although PAX8 positivity has been reported in a relevant proportion (6\%-18\%) of peritoneal malignant mesotheliomas, usually the staining is weak and focal. ${ }^{18-20}$ The most reliable recently discovered markers for the diagnosis of mesothelioma are loss of BRCA-associated protein 1 (BAP1) by IHC and deletion of $\mathrm{p} 16$ by fluorescence in situ hybridisation; therefore, these markers can certainly help in this differential diagnosis. ${ }^{21-24}$

\section{Mucinous adenocarcinoma: ovarian primary versus metastatic} The most difficult differential diagnosis in the ovarian cancer field concerns mucinous tumours since both morphological and immunophenotypical features are shared between primary and metastatic tumours. Indeed, macroscopic features and clinical correlation remain fundamental for a correct diagnosis (table 1). ${ }^{2}$ Immunohistochemically, there is a significant overlap in the immunophenotypes between primary mucinous ovarian carcinoma and metastatic gastrointestinal carcinoma. Typically, CK20, CDX2 and SATB2 are expressed by colorectal adenocarcinoma and show an intense and diffuse pattern. Notably, they are negative, or only focal and weak, and in any case less intense and diffuse than CK7, in primary ovarian carcinomas, with the only exception being the rare intestinal-type mucinous ovarian tumours originating from ovarian teratomas. ${ }^{25}$

In primary ovarian mucinous tumours, besides cytokeratin 7 (CK7) and CA125, PAX8 is expressed in 65\% of cases, but not in colorectal adenocarcinomas. ${ }^{26}$ Notably, CA125 is not ovarian specific; even breast, lung, pancreas, cervix and uterine carcinomas and mesothelioma may be positive. Therefore, though $\mathrm{CK} 20+/ \mathrm{CK} 7-$ is prototypical for metastatic adenocarcinomas from the lower intestinal tract and this immunoprofile can be definitive for correct diagnosis, often it is necessary to resort to lineage-specific markers PAX8 and SATB2, both highly specific but defectively sensitive. ${ }^{26} 27$ In this situation, ER and PR are of limited value since they are negative in both intestinal-type primary and metastatic carcinomas, whereas CDX2 is a site-unspecific marker of intestine differentiation (see below). ${ }^{28-30}$

Finally, and uniquely, SMAD4 (DPC4) is lost in half of all pancreatic cancers. ${ }^{3132}$ A summary of the many tissue-specific IHC markers that may help in the differential diagnosis of tumours from various sites is reported in table 2 and representative pictures in figure $2 .^{21233-36}$

\section{Small cell carcinoma of hypercalcaemic type versus other mimics}

Small cell carcinoma of hypercalcaemic type (SCC-HT), a rare but aggressive tumour, can simulate mainly HGSC and adult granulosa cell tumour, but typically arises in children and young women, and is associated with hypercalcaemia. Similarly to HGSC, SCC-HT also demonstrates IHC positivity for $\mathrm{p} 53$ and WT1. Characteristically, SCC-HT shows calretinin positivity, similar to granulosa cell tumours, although focal and weak. Morphologically, SCC-HT is reminiscent of small cell lung carcinoma and present hyperchromatic, ungrooved nuclei, with frequent mitoses. Recently, it has been shown that SMARCA4 is specifically mutated in over $90 \%$ of SCC-HT, and this genetic aberration produces a loss of SMARCA4 (BRG1) protein expression $^{37}$ that can be used as a valid adjunct in the differential diagnosis of SCC-HT.

\section{Clear cell carcinoma versus yolk sac tumour}

CCC and yolk sac tumour share many morphological features, such as glycogen-rich clear cells with atypical nuclei with occasional nucleoli and infrequent mitoses, papillary growth, loosened oedematous pattern, hyaline globules and HNF1 $\beta$ positivity. Importantly, CCC often arises in a background of endometriosis, or clear cell adenofibroma, and it is usually positive for AMACR, CK7, EMA and Napsin A and negative for AFP and glypican 3, as opposed to yolk sac tumour. ${ }^{33} 3839$ Moreover, SALL4, a specific and sensitive marker for germ cell tumours, may reliably distinguish yolk sac tumour since it stains intensely and diffusely almost all cases analysed, but it is negative or very rarely only focal in CCC. ${ }^{39} 40$

\section{LOOKING AT THE FALLOPIAN TUBE}

\section{Serous tubal intraepithelial carcinoma versus other mimics}

Ever-growing evidence identifies serous tubal intraepithelial carcinoma (STIC) as the most likely precursor lesion of HGSC. To solve doubtful tubal lesions, a diagnostic algorithm, which includes marked cytological atypia, Ki-67 proliferation index and p53 IHC, has been proposed. ${ }^{41} 42$ Based on this algorithm, STICs are intramucosal tubal lesions that combine cytological atypia with Ki-67 proliferation index $>10 \%$ and p53 IHC mutant pattern.

\section{LOOKING AT THE UTERUS}

\section{Endometrial carcinoma subtyping}

Since 1983, endometrial carcinoma has been proposed to follow a dualistic pathogenetic model. ${ }^{43}$ Endometrioid carcinoma is the prototypical type I endometrial carcinoma, whereas type II tumours include USC, CCC, malignant mixed Müllerian tumour and undifferentiated carcinoma. Their distinction, usually elementary, has important implications for pathobiology and treatment. In some instances, such as glandular-cribriform USC, papillary endometrioid carcinoma and endometrioid carcinoma with clear cells, their discrimination may be difficult and IHC aids may be necessary (table 3). Endometrioid carcinomas are usually ER and PR positive, whereas USC and CCC are negative. ${ }^{44}$ In addition, USC harbours TP53 mutation and a mutant 


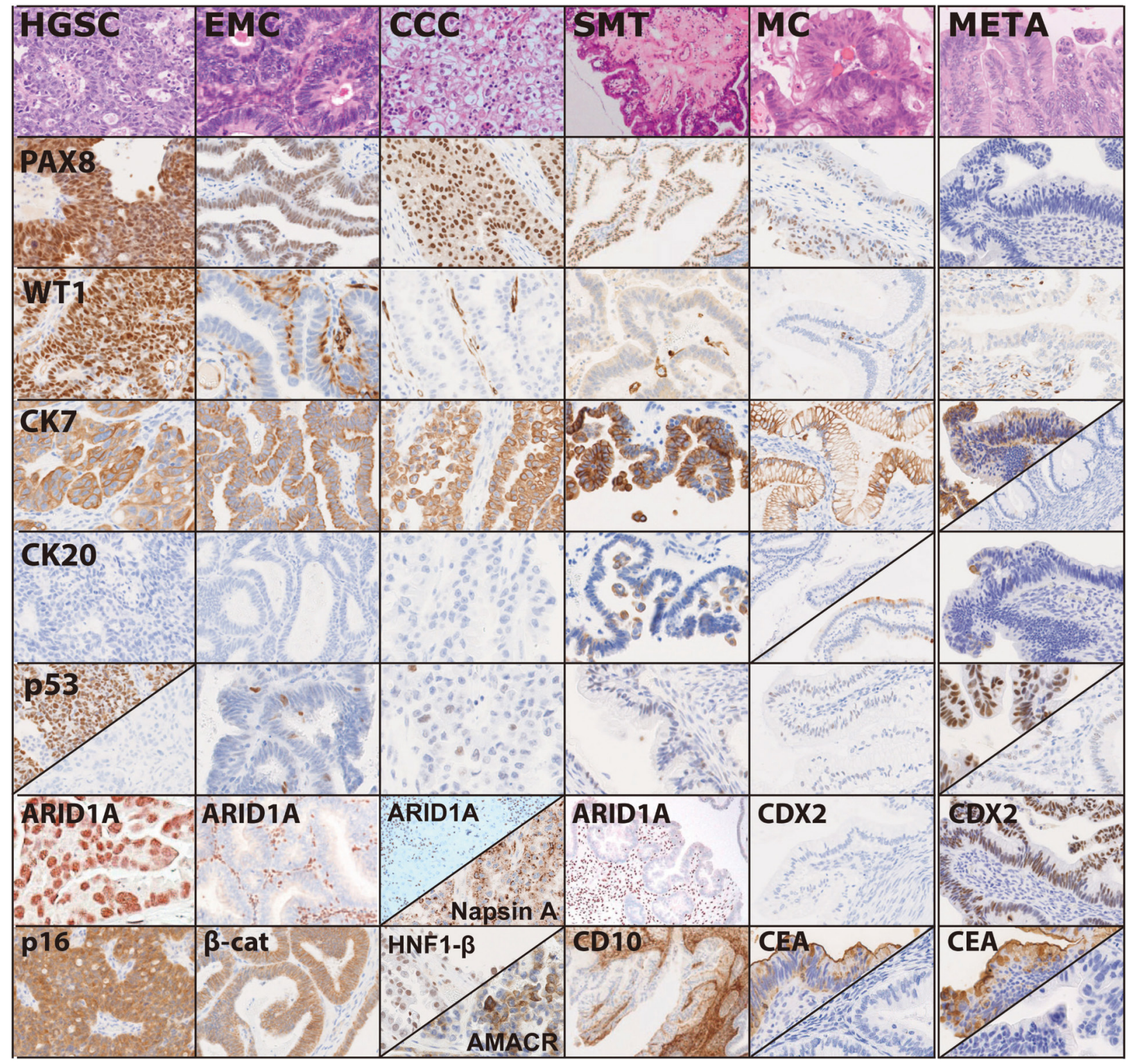

Figure 2 Representative images of the most helpful immunostainings used in the differential diagnosis of ovarian epithelial tumours. $\beta$-cat, $\beta$-catenin; CCC, clear cell carcinoma; EMC, endometrioid carcinoma; HGSC, high-grade serous carcinoma; MC, mucinous carcinoma; META, metastatic mucinous carcinoma; SMT, seromucinous tumour.

Table 3 Immunohistochemical (IHC) markers in the differential diagnosis of endometrial carcinomas

\begin{tabular}{|c|c|c|c|c|c|c|c|c|c|c|c|}
\hline & vim & ER & PR & ARID1A & $\beta$-cat & AMACR & HNF1 $\beta$ & Napsin A & p53 & p16 & Specific \\
\hline \multicolumn{12}{|c|}{ Endometrial tumours } \\
\hline EMC & + & + & + & $M>w t$ & $M>w t$ & $+/-$ & - & - & $w t>M$ & $-1+$ & PTEN and MMR loss \\
\hline $\mathrm{CCC}$ & + & - & - & wt & wt & $-1+$ & + & $+1-$ & $w t>M$ & - & MMR loss \\
\hline UC & - & - & - & wt & $M>w t$ & NA & - & NA & $M>w t$ & $-1+$ & E-cadherin and MMR loss \\
\hline MMMT & + & $-/+$ & $-1+$ & $w t>M$ & wt & NA & - & NA & M & + & $\begin{array}{l}\text { WT1, desmin, CD10, h- caldesmon, } \\
\text { myogenin, S100, Myo-D1. Biphasic pattern }\end{array}$ \\
\hline
\end{tabular}

ARID1A M consists in the loss of IHC staining, while ARID1A wt is nuclear staining; p53 M corresponds to intense and diffuse positivity in $\geq 60 \%$ of cells or complete negativity; p53 wt is the presence of rare cells weakly positive or positivity in $<60 \%$ of cells. $\beta$-cat $\mathrm{M}$ is nuclear positivity; $\beta$-cat wt is cytoplasm, membrane positivity or negativity. $\beta$-cat, $\beta$-catenin; CCC, clear cell carcinoma; EMC, endometrioid carcinoma; M, mutant pattern; MMMT, malignant mixed Müllerian tumour; MMR, DNA mismatch repair genes; NA, not available; UC, undifferentiated carcinoma; USC, uterine serous carcinoma; wt, wild-type pattern. 


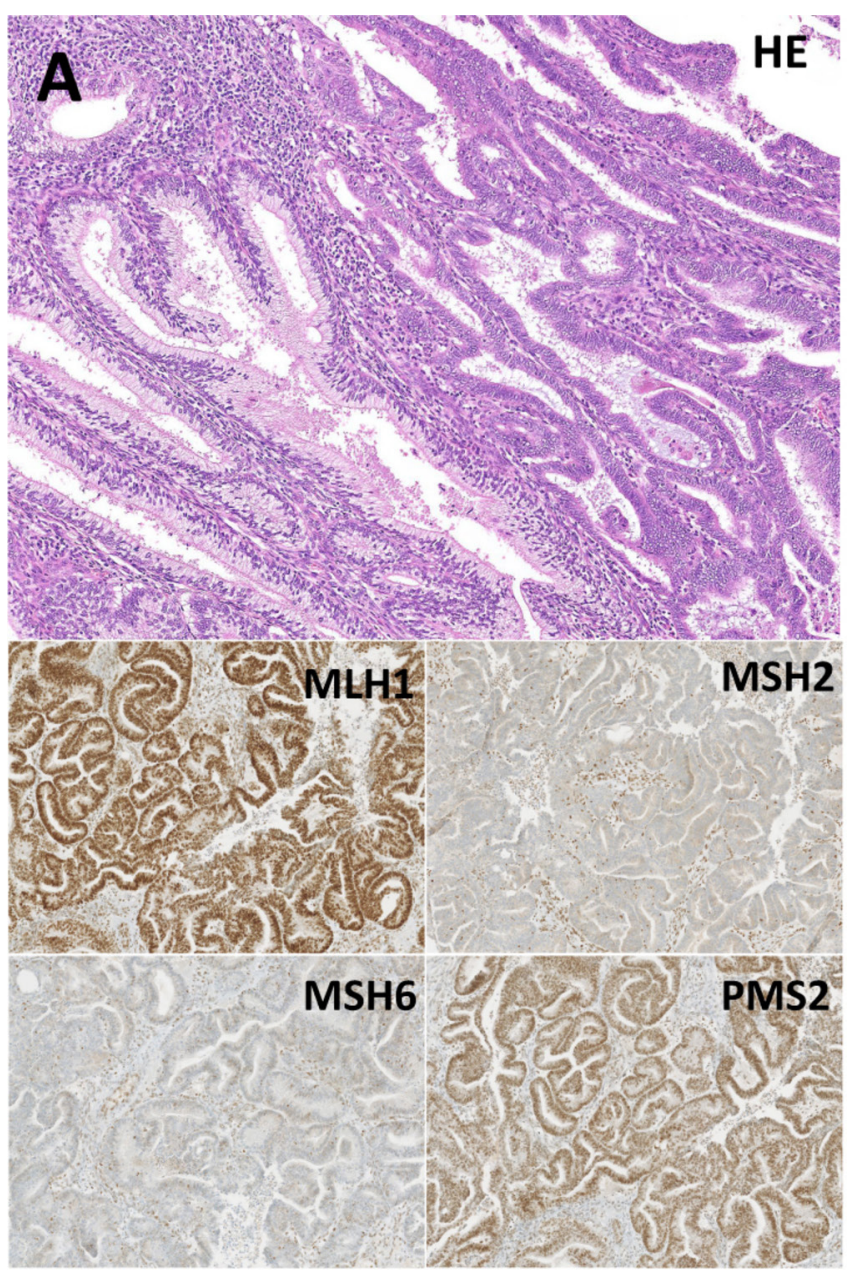

B

MMR protein expression and mutations

\begin{tabular}{|l|c|c|c|c|c|}
\hline Mutation & $\begin{array}{c}\text { MLH1 } \\
\text { IHC }\end{array}$ & $\begin{array}{c}\text { MSH2 } \\
\text { IHC }\end{array}$ & $\begin{array}{c}\text { MSH6 } \\
\text { IHC }\end{array}$ & $\begin{array}{c}\text { PMS2 } \\
\text { IHC }\end{array}$ & MSI \\
\hline MLH1 & - & + & + & - & $\mathrm{H}$ \\
\hline MSH2 & + & - & - & + & $\mathrm{H}$ \\
\hline MSH6 & + & + & - & + & $\mathrm{H} / \mathrm{L}$ \\
\hline PMS2 & + & + & + & - & $\mathrm{H}$ \\
\hline EPCAM & + & - & - & + & $\mathrm{H}$ \\
\hline
\end{tabular}

Figure 3 (A) Endometrioid carcinoma associated with Lynch syndrome shows prominent peritumoural lymphocytes (H\&E staining, upper picture), and retention of MLH1 and PMS2 and loss of MSH2 and MSH6 by immunohistochemistry. (B) Correlation between mutations, immunohistochemical (IHC) expression of mismatch repair (MMR) genes and microsatellite instability (MSI) status in Lynch syndrome-associated cancers. HE, haematoxylin-eosin; +, positive nuclear staining; -, negative nuclear staining; $H$, high; $L$, low.

p53 IHC pattern (see above), whereas endometrioid carcinoma and CCC are usually TP53 wild-type. ${ }^{43}$ Conversely, endometrioid carcinoma and CCC harbour ARID1A,CTNNB1 and DNA mismatch repair gene (MMR) mutations and a mutant IHC pattern (figure 3). ${ }^{45}$ Interestingly, p16 tends to be strongly and diffusely positive in USC, only focal in endometrioid carcinoma and negative in CCC. Finally, diffuse HNF1及 and Napsin
A positivity favours CCC. ${ }^{46}$ Notably, also Arias-Stella reaction and some endometrioid carcinomas may express HNF1及.

Undifferentiated carcinoma deserves its own chapter. Characteristics of this entity include the IHC negativity or only focal positivity for CKs (AE1/AE3, 8, 18, 8/18), vimentin, EMA, ER, PR, chromogranin, synaptophysin, E-cadherin and CTNNB1, TP53 and MMR gene mutation in about 30\%, 30\% and 50\% of cases, respectively. ${ }^{547}$ Recently, McCluggage's group reported CD34 IHC expression in 29\% of undifferentiated carcinomas and found a relative frequency of loss of IHC expression of SMARCA4 and SMARCA2, members of the SWI/SNF chromatin-remodelling complex. ${ }^{48} 49$

Malignant mixed Müllerian tumour (ie, carcinosarcoma) is an aggressive biphasic tumour composed of both carcinomatous and sarcomatous cells. Usually, morphological features are sufficient to diagnose this entity. In rare cases, high p53/WT1 and low ER/PR expression are helpful for differentiating malignant mixed Müllerian tumours from endometrioid carcinomas with spindle cell differentiation. ${ }^{50} 51$

\section{Endometrial hyperplasia versus endometrioid intraepithelial neoplasia versus endometrioid carcinoma}

The new classification that defines endometrioid intraepithelial neoplasia (EIN, also known as atypical hyperplasia) as a monoclonal mutated precursor of endometrioid carcinoma permits the use of IHC markers of early mutation to tell endometrial hyperplasia apart from EIN. To this end, nuclear $\boldsymbol{\beta}$-catenin and MLH1 or PTEN loss have been demonstrated useful. ${ }^{52}{ }^{53}$ In addition, several authors have reported the utility of PAX2 loss to decipher tricky EIN cases. ${ }^{54-57}$

Furthermore, diagnostic problems in distinguishing EIN from well-differentiated endometrioid carcinoma have long been studied, but there still is no reliable marker, other than the careful and strict implementation of histopathological criteria specifically defined for diagnosis of endometrioid intraepithelial neoplasias. ${ }^{58}$ On the other hand, although both lesions show PTEN loss, additional loss of ARID1A and increased Ki-67 proliferation index may warn for endometrioid carcinoma. ${ }^{59} 60$

\section{Müllerian adenosarcoma versus endometrial polyp}

The peculiar phyllode-like growth pattern, periglandular stromal hypercellularity and mild-moderate atypia characterise Müllerian adenosarcoma. When morphological features are not conclusive, Ki-67 IHC may help since adenosarcoma shows a zonal periglandular increase of $\mathbf{K i}-67$, in contrast to the endometrial polyp and atypical polypoid adenomyoma. ${ }^{61}$

\section{Endometrial stromal sarcoma}

The classification of endometrial stromal sarcomas has been refined on the basis of the molecular characteristics recently identified. The most recent WHO classification identifies three different sarcomas likely derived from endometrial stromal cells: low-grade endometrial stromal sarcoma, high-grade endometrial stromal sarcoma and undifferentiated uterine sarcoma. Indeed, low-grade and high-grade endometrial stromal sarcomas are characterised by a simple karyotype and distinctive pathognomonic chromosomal translocations, resulting in JAZF1SUZ12 and YWHAE-NUTM2A/B gene fusions, respectively. ${ }^{62-64}$ However, molecular testing is not currently standard practice; therefore, immunophenotype is helpful in the differential diagnosis. Typically, low-grade endometrial stromal sarcomas show diffuse immunopositivity for CD10, ER and PR. ${ }^{65}$ In contrast, high-grade endometrial stromal sarcomas lack CD10, ER and PR 
Table 4 Immunohistochemical markers in the differential diagnosis of endometrial mesenchymal tumours

\begin{tabular}{|c|c|c|c|c|c|c|c|c|c|}
\hline & vim & ER & PR & p53 & p16 & CD10 & desm & h-cald & Specific \\
\hline \multicolumn{10}{|c|}{ Endometrial mesenchymal tumours } \\
\hline LGESS & + & + & + & wt & - & + & - & - & JAZF1-SUZ12 fusion \\
\hline HGESS & + & - & - & wt & - & - & - & - & $\begin{array}{l}\text { YWHAE-NUTM2A/B fusion, } \\
\text { cyclin D1, c-Kit, } \\
\text { Ki-67 high }\end{array}$ \\
\hline LM & + & + & + & wt & - & - & + & + & Actin \\
\hline LMS & + & $-1+$ & $-1+$ & $M>w t$ & + & - & + & + & Ki-67 high \\
\hline
\end{tabular}

p53 M corresponds to intense and diffuse positivity in $\geq 60 \%$ of cells or complete negativity; p53 wt is the presence of rare cells weakly positive or positivity in $<60 \%$ of cells. desm, desmin; h-cald, h-caldesmon; HGESS, high-grade endometrial stromal sarcoma; LGESS, low-grade endometrial stromal sarcoma; LM, leiomyoma; LMS, leiomyosarcoma; M, mutant pattern; wt, wild-type pattern.

expression and display a strong nuclear positivity for cyclin D1 and membranous/cytoplasmic reactivity for c-Kit. ${ }^{65-67}$ Finally, undifferentiated uterine sarcomas include a heterogeneous group of aggressive neoplasias harbouring complex karyotype and showing marked pleomorphism, necrosis and mitotic activity and variable expression of CD10, ER and PR. ${ }^{68}$ Undifferentiated uterine sarcomas should be diagnosed only after exclusion of the most common mimics: leiomyosarcoma, carcinosarcoma, undifferentiated carcinoma, rhabdomyosarcoma and diffuse large B-cell lymphoma. ${ }^{69}$

\section{Endometrial stromal versus uterine smooth muscle tumour}

Some leiomyomas show morphological similarity to endometrial stromal tumours, particularly cellular leiomyomas. Smooth muscle tumours, as opposed to low-grade endometrial stromal sarcoma, usually express desmin and h-caldesmon but not CD10 ${ }^{70-73}$ (table 4).

\section{Leiomyosarcoma versus leiomyoma}

Difficult smooth muscle tumours may necessitate taking advantage of IHC because diffuse $\mathrm{p} 53$ and $\mathrm{p} 16$ and a high Ki-67 proliferation index favour a leiomyosarcoma; however, some smooth muscle tumours with uncertain malignant potential and some leiomyomas rarely show overlapping patterns. ${ }^{74-76}$

\section{Cervical squamous neoplasia versus benign mimics}

The main problem in cervical pathology is to distinguish intraepithelial lesions from their innocuous mimics, such as reactive and metaplastic squamous changes, atrophy and cytological atypia due to cautery artefact. In this context, p16 IHC positivity is a surrogate marker for high-risk human papilloma virus (HPV) infection. Normally, p16 protein is inhibited by $\mathrm{Rb}$ negative feedback, which is interrupted during HPV infection. In particular, E7 viral oncogene constitutively inactivates $\mathrm{Rb}$ pathway through sequestration of $\mathrm{pRb}$, hence, HPV integration in cycling cervical cells results in a block-type strong and diffuse p16 positivity. ${ }^{77}$ Notably, p16 IHC positivity specific for highrisk HPV lesions is strong continuous staining, both nuclear and cytoplasmic, involving at least the basal third of the epithelial full thickness (ie, block staining). ${ }^{77}$ This $\mathrm{p} 16$ block positivity is found in the majority of high-grade squamous intraepithelial lesions (HSILs) and only in one-third of low-grade squamous intraepithelial lesions (LSILs). In particular, although HSIL with severe dysplasia (corresponding to CIN-3) is almost invariably positive for $\mathrm{p} 16$, nearly one-third of the HSIL spectrum's lower extremity (corresponding to $\mathrm{CIN}-2$ ) has been reported to be negative for $\mathrm{p} 16 .^{7879}$ Importantly, the new recommendations for squamous lesions of the lower anogenital tract (LAST) include the use of p16 immunostaining in lesions morphologically doubtful for CIN-2, and this practice could jeopardise diagnosis of these p16-negative HSILs, resulting in underdiagnosis as LSIL. ${ }^{77}$ Next related issue is whether $\mathrm{p} 16$-negative CIN-2 behave like p16-positive ones. So far, a definitive answer to this question is missing, and the studies on this topic are limited and contradictory. However, recent studies on intermediate dysplastic lesions found that p16-positive lesions are more likely to persist or progress behaving like HSILs, whereas p16-negative lesions tend to regress as well as LSILs, providing evidence of the clinical 'correctness' of LAST recommendations. ${ }^{78} 80$

In addition, SILs show an increased proliferation index with Ki-67. Along with p16 positivity, Ki-67 may also reliably assist the differential diagnosis between HSIL and LSIL, whereby full-thickness proliferation favours HSIL. Clearly, appropriate orientation of the epithelium is necessary in order to prevent misinterpretation.

Recently, the squamocolumnar junction (SCJ) cells have gained increasing interest as possible cells of origin of SILs, hence, the IHC expression of the SCJ proteins (ie, CK7, CK17, MMP7 and p63) has been studied in SILs. ${ }^{81} 82$ Interestingly, it has emerged that SCJ markers are strongly and diffusely expressed in HSILs, but either negative or patchy in LSILs, so that they may be used as adjunct IHC markers in distinguishing between HSIL and LSIL. This is particularly true for CK7 since it is widely used. In addition, CK7 expression has been correlated with an increased risk of LSIL progression and accordingly proposed as a risk stratifier. $^{83-85}$

\section{Cervical glandular neoplasia versus benign mimics}

Likewise, endocervical adenocarcinoma in situ (AIS) must be distinguished from potential innocuous mimics such as reactive and reparative glandular changes, tubal metaplasia, microglandular hyperplasia and endometriosis. ${ }^{86}$ Immunohistochemically, AIS shows increased Ki-67 and diffuse p16 and mCEA, but negative vimentin and ER. Conversely, p16 in benign lesions tends to be negative or focal, and $\mathrm{Ki}-67$ proliferation index is low $(<10 \%){ }^{8687}$

\section{Endocervical versus endometrial adenocarcinoma}

The therapeutic approach for endometrial carcinoma and endocervical-type adenocarcinoma is different; therefore, indicating the cancer origin is of extreme importance, but may be tricky based only on morphology, particularly in curettage samples. Morphological characteristics though may be of guidance. Typically, endocervical adenocarcinoma of the usual type is morphologically characterised by (1) angulated and branching glands, (2) nuclear crowding and pseudostratification, (3) nuclear hyperchromasia and marked atypia, and (4) numerous basal apoptotic bodies and mitotic figures, usually apical. Moreover, 
Table 5 Immunohistochemical markers in the differential diagnosis of endometrial carcinomas versus endocervical adenocarcinomas

\begin{tabular}{|c|c|c|c|c|c|c|c|c|c|}
\hline & vim & ER & PR & p53 & p16 & HNF1及 & WT1 & CEA & Specific \\
\hline \multicolumn{10}{|l|}{ Endometrial carcinoma } \\
\hline EMC & + & + & + & $w t>M$ & $-1+$ & - & - & - & PTEN and MMR loss \\
\hline USC & + & - & - & $\mathrm{M}$ & + & - & - & - & \\
\hline $\mathrm{CCC}$ & + & - & - & $w t>M$ & - & + & $-1+$ & - & $\begin{array}{l}\text { HNF1 } \beta \text {, Napsin A } \\
\text { MMR loss }\end{array}$ \\
\hline UC & - & - & - & $M>w t$ & $-/+$ & - & - & - & E-cadherin and MMR loss \\
\hline MMMT & + & $-1+$ & $-1+$ & M & + & - & $+1-$ & - & $\begin{array}{l}\text { Myogenin, S100, Myo-D1. Biphasic } \\
\text { pattern }\end{array}$ \\
\hline \multicolumn{10}{|c|}{ Endocervical adenocarcinoma } \\
\hline AIS & - & $-l+$ & $-1+$ & wt & + & - & - & + & $\mathrm{HPV}+$ \\
\hline EAC, usual type & - & $-1+$ & $-1+$ & wt & + & $-1+$ & - & + & $\mathrm{HPV}+$ \\
\hline MC, gastric type & - & - & - & $M>w t$ & $-1+$ & + & - & $+1-$ & MUC6, HIK1083, STK11 mutation \\
\hline MC, intestinal type & - & - & - & & + & - & - & + & $\mathrm{CDX} 2, \mathrm{HPV}+$ \\
\hline Mesonephric carcinoma & $+1-$ & $-1+$ & - & wt & $-1+$ & $-1+$ & - & - & TTF1, GATA3, calretinin \\
\hline EMC & $-1+$ & $-1+$ & $-1+$ & wt & $\begin{array}{l}-1 \\
+\end{array}$ & NA & - & $+/-$ & $\mathrm{HPV}+$ \\
\hline Serous carcinoma & - & $-1+$ & $-1+$ & $w t>M$ & + & NA & $-1+$ & + & $\mathrm{HPV}+$ \\
\hline Clear cell carcinoma & & $-1+$ & - & $w t>M$ & $+1-$ & + & $-1+$ & - & Napsin A, PIK3CA mutation \\
\hline
\end{tabular}

p53 $\mathrm{M}$ corresponds to intense and diffuse positivity in $\geq 60 \%$ of cells or complete negativity; $\mathrm{p} 53$ wt is the presence of rare cells weakly positive or positivity in $<60 \%$ of cells. AIS, endocervical adenocarcinoma in situ, of usual type; CCC, clear cell carcinoma; EAC, endocervical adenocarcinoma; EMC, endometrioid carcinoma; HPV+, human papilloma virus infection in situ hybridisation; M, mutant pattern; MMMT, malignant mixed Müllerian tumour; MMR, DNA mismatch repair genes; UC, undifferentiated carcinoma; USC, uterine serous carcinoma; wt, wild-type pattern.

endocervical adenocarcinoma is often associated with concurrent AIS and squamous lesions. ${ }^{88} 89$ On the other hand, endometrioid endometrial carcinoma is characterised by (1) predominant tubular architecture, (2) cells with scant cytoplasm and large vesicular nuclei, not pseudostratified, (3) presence of stromal foamy histiocytes and (4) squamous metaplasia. Commonly, EIN accompanies endometrioid carcinoma.

The general IHC panel for this situation includes ER, PR and vimentin strongly positive in endometrial carcinoma, and $\mathrm{p} 16$, mCEA and HPV in situ hybridisation diffusely positive in endocervical adenocarcinoma of the usual type.

Notably, p53 positivity in a cervical carcinoma strongly suggests against common cervical carcinomas, that is, squamous carcinoma or endocervical adenocarcinoma of usual type, and in all cases, a diagnosis of a secondary serous carcinoma should be considered and ruled out. ${ }^{86}$ However, p53 positivity is present in a consistent percentage of endocervical adenocarcinoma of gastric type or serous carcinoma of the cervix, though the latter variant is exceedingly rare. In addition, HNF1 $\beta$ positivity is not restricted to CCC, but it has been also reported in gastric-type and mesonephric carcinomas. ${ }^{90-92}$ The IHC markers useful for the diagnosis of endometrial adenocarcinoma and special-type endocervical adenocarcinomas are reported in table 5.

\section{ROLE OF IMMUNOHISTOCHEMISTRY IN THE DIAGNOSIS OF SYNDROMIC GYNAECOLOGICAL CANCERS}

Both ovarian and endometrial carcinomas can be an indication of genetically inherited syndromes.

Two main hereditary cancer syndromes are known that predispose to cancers occurring in the gynaecological tract, namely Lynch and hereditary breast and ovary cancer syndromes.

Lynch syndrome is due to germline mutations in the MMR system, including MLH1, MSH2, MSH6 and PMS2 genes, and in EPCAM gene that cause high microsatellite instability (MSI) and increase the risk of endometrioid, clear cell and undifferentiated carcinomas. Impaired DNA mismatch repair results in alterations to hypermutable short repetitive sequences in the genome (microsatellites) and its detection conventionally performed by IHC analysis of the aforementioned MMR proteins. ${ }^{93}$ Lynch syndrome is an under-recognised entity, responsible for $5 \%$ of endometrial cancers and $1 \%$ of ovarian cancer cases, but high MSI is found in more than $30 \%$ of endometrial and around 3\% of ovarian carcinomas due to somatic mutations and MLH1 promoter methylation. The pathologist plays a fundamental role in identifying cancers harbouring high MSI. In fact, there are some histological clues suggesting Lynch syndrome: prominent peritumoural lymphocytes, increased lymphocytes ( $>42$ per 10 high-power fields) located within the boundary of tumour cell nests or glands, and tumour heterogeneity, defined as juxtaposed distinct tumour populations constituting more than $10 \%$ of the tumour volume, along with an undifferentiated component. ${ }^{94-96}$ To confirm Lynch syndrome, the pathologist should first apply IHC for MLH1, MSH2, MSH6 and PMS2 (figure 3), which is extremely reliable, then request MSI analysis by PCR. In identifying the presence of MMR germline mutations in EMCs, the sensitivity ranges from $77 \%$ to $100 \%$ for MSI assay by PCR and from $86 \%$ to $100 \%$ for MMR IHC, whereas the specificity ranges from $38 \%$ to $81 \%$ and from $48 \%$ to $81 \%$, respectively. ${ }^{97-99} \mathrm{~A}$ study by McConechy et al showed $93 \%$ of concordance between the two methods in EMCs and stated that the two methods 'are equivalent' in detecting MMR defects. ${ }^{97}$ Importantly, MMR IHC has low cost, fast turnaround time, identifies which gene is mutated and can be performed on routine tissue material. ${ }^{97}$ On the other hand, MSI assay may identify MMR defects that do not affect IHC stainings or that are in genes not tested by IHC, such as MSH3 and PMS1. ${ }^{100}$ Moreover, MSI assay is unambiguous, easy to read, highly reproducible and requires less material (one section vs four sections). However, a recent paper found that MSI assay in EMCs, when compared with colorectal cancers, has a slightly higher false-negative rate than IHC due to the high prevalence of one-nucleotide shifts that can be missed by MSI assay. $^{101}$

Hereditary breast and ovary cancer syndrome is due to germline mutations in breast cancer 1 (BRCA1) and BRCA2 genes. 


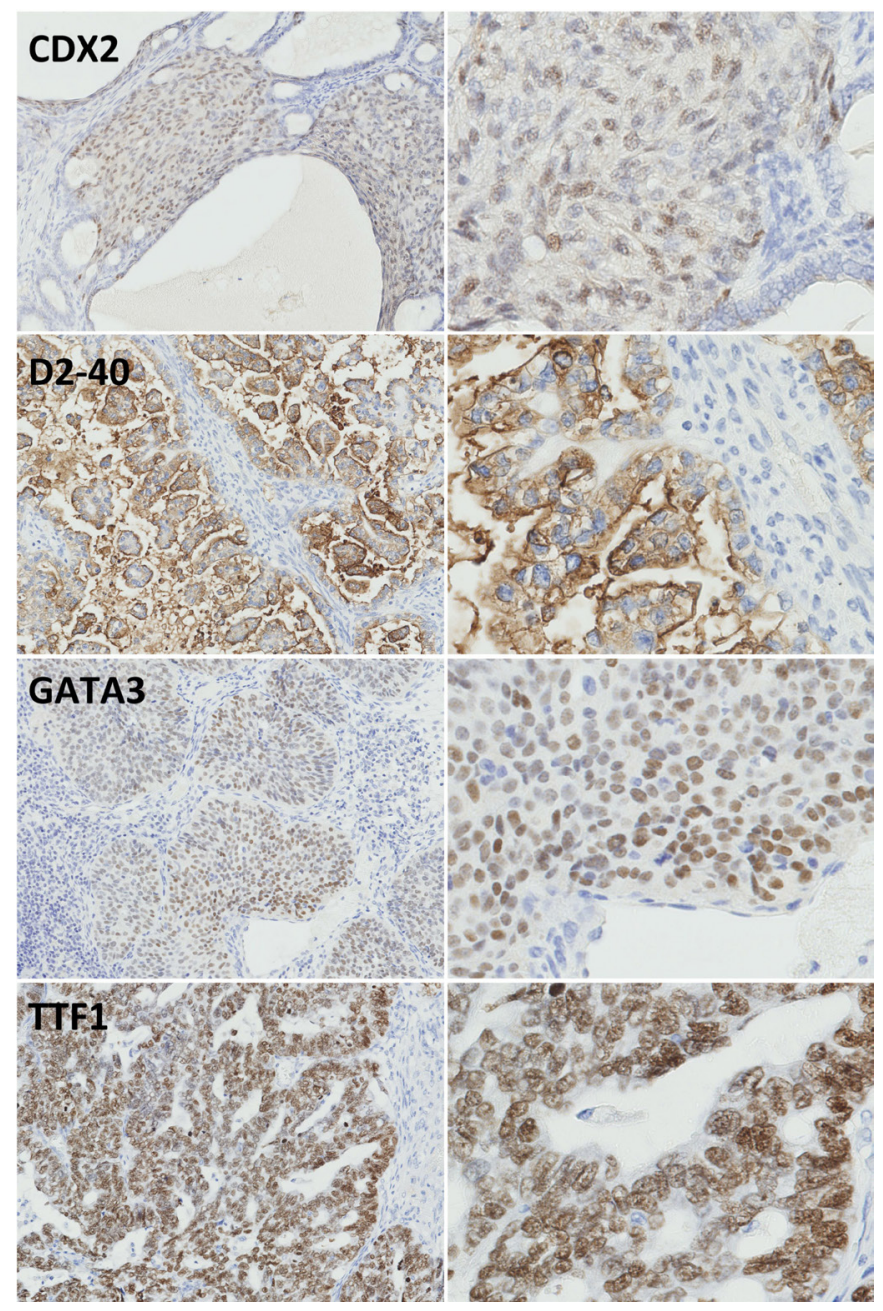

Figure 4 Examples of unexpected immunohistochemical stainings in gynaecological neoplasias are shown: CDX2 is weakly positive in an endometrioid carcinoma, mainly in the morular metaplasia; D2-40 positivity in the presented ovarian clear cell carcinoma is intense and apical; a relevant proportion of squamous cell carcinomas of the cervix is convincingly GATA3 positive, but usually focal and weak; and TTF1 can be remarkably positive in endometrioid carcinoma, as shown in the last panels (left panels, magnification $\times 200$; right panels, magnification $\times 630$ ).

These germline mutations cause about $5 \%-10 \%$ of all ovarian carcinomas and a small minority of endometrial carcinomas. Specifically, women with mutated BRCA1 and BRCA2 have a $40 \%$ and a $20 \%$ lifetime risk of developing ovarian carcinoma, respectively, and a 2\%-3\% lifetime risk of developing endometrial carcinoma. ${ }^{102}$ On the other hand, it is known that about $12 \%$ of ovarian HGSCs have disruption of the BRCA pathway due to somatic events, either BRCA1/BRCA2 mutation or BRCA1 promoter methylation. Histologically, cancers with $B R C A$ mutations are typically of high-grade histology, virtually all HGSC in the ovary and either serous or clear cell carcinomas in the endometrium. BRCA-mutated HGSCs show a SET growth pattern (solid, pseudoendometrioid and transitional-like) and, specifically, cases with a BRCA1 mutation also present increased tumour intraepithelial lymphocytes, brisk mitotic indexes and necrosis. ${ }^{103}$ In addition, a micropapillary infiltrative pattern of metastatic HGSC with BRCA germline mutations is more frequently seen in BRCA1-mutated cases and has been associated with poor prognosis. ${ }^{104}$
Currently, much effort is devoted to developing BRCA1 and BRCA2 antibodies able to identify patients with compromised BRCA pathway with promising results, but they are not translated yet into daily practice. ${ }^{105} 106$ Therefore, BRCA testing is mainly based on molecular techniques, as real-time PCR or sequencing, and dedicated to women with a familial history. BRCA pathway alterations are known to cause an improved response to platinum-based therapy and to render patients eligible to PARP (ie, poly(ADP)-ribose polymerase) inhibitors. ${ }^{107-109}$

Given that mutations causing these syndromes and related somatic molecular alterations are responsible for specific sensitivity or resistance to therapy, it is plausible that they will shortly drive therapeutic choices; however, our knowledge is still lacking. ${ }^{110}$

\section{UNEXPECTED IMMUNOSTAININGS}

Any immunohistochemical marker is specific until proven otherwise; as a consequence, some organ-associated immunohistochemical markers, not properly Müllerian, have also been described in gynaecological tumours (figure 4).

CDX2 is a homeobox transcription factor that is expressed in intestinal epithelial cells and is used in diagnostic pathology as a marker of intestinal carcinoma, mainly colonic, but also oesophageal, gastric and biliopancreatic adenocarcinoma. Interestingly, the CDX2 expression has been identified in lung and bladder carcinomas and considered as a marker of intestinal differentiation. ${ }^{29}$ Coherently, in gynaecological neoplasms, the CDX2 expression has been well characterised in ovarian mucinous adenocarcinoma (previously called intestinal type) where it is positive between $36 \%$ and $94 \%$ of cases, as well as in $39 \%$ cervical adenocarcinoma, especially of intestinal type. ${ }^{30} 3435111-113$

Moreover, CDX2 positivity in endometrial carcinomas ranged from $6 \%$ to $44 \%$. Interestingly, two studies reported that CDX2 expression in endometrial endometrioid carcinoma is associated with morular differentiation. ${ }^{114} 115$ Analogously, 0\% to 30\% of ovarian endometrioid carcinomas have been reportedly positive for CDX2.

D2-40 (also known as podoplanin) is a mesothelial and lymphatic endothelial marker. Besides gynaecological adenomatoid tumours, peritoneal mesotheliomas and vascular tumours, a variable proportion of ovarian carcinomas have shown immunopositivity for podoplanin, depending on the histotype. ${ }^{116117}$ Specifically, 10\%-65\% of serous carcinomas, $0 \%-33 \%$ of endometrioid carcinomas, $0 \%-16 \%$ of mucinous carcinomas and $0 \%-55 \%$ of CCCs have been reported positive for podoplanin, and based on one study, the latter showed stronger positivity. ${ }^{116-119}$ Eventually, podoplanin may help to discriminate dysgerminoma since it is invariably positive in dysgerminoma cells but negative in the other ovarian germ cell tumours. ${ }^{119}$

GATA3 has a pivotal role in the embryogenesis and differentiation of the breast, urothelial and T cells, and as such, its IHC expression is commonly used as a surrogate marker for mammary and urothelial derivation in neoplasias of unknown origin. In gynaecological pathology, GATA3 has shown a weak and focal positivity in endocervical, endometrial and ovarian adenocarcinoma in up to $18 \%, 23 \%$ and $10 \%$, respectively. ${ }^{120121}$ Among special gynaecological tumours, the majority of Brenner tumours are diffusely GATA3 positive, similarly to urothelial carcinoma, but only $50 \%$ of transitional cell carcinomas. ${ }^{122}$ Alike, between $26 \%$ and $60 \%$ of squamous cell carcinomas are remarkably positive for GATA3, but only focally. ${ }^{120} 123$ Of note, all gestational trophoblastic tumours 
and almost the totality of mesonephric carcinomas (95\%) express GATA3 consistently. ${ }^{124} 125$

Thyroid transcription factor-1 (TTF1) is a lineage-specific marker expressed in lung and thyroid parenchyma and primarily used as specific immunomarker of lung and thyroid carcinomas. Recently, TTF1 has been found expressed in a minority of ovarian (3\%-39\%), endometrial (2\%-23\%) and cervical adenocarcinomas $(4 \%) .{ }^{126-130}$

\section{CONCLUSIONS}

This short review intends to provide an updated overview of the essential IHC markers currently used in the diagnostics of gynaecological diseases along with their molecular rationale. Over the last decade, there have been unpredictable advances in the understanding of the molecular pathogenesis of gynaecological malignancies. It is advised that this knowledge becomes the foundation for a molecularly oriented therapeutic approach to improve the outcome and reduce the side effects of patients with gynaecological cancer.

Correction notice This article has been corrected since it was published Online First. The corresponding details has been updated to include Prof Ayse Ayhan.

Handling editor Dhirendra Govender.

Contributors EK and AA contributed equally to this manuscript.

Competing interests None declared.

Provenance and peer review Not commissioned; externally peer reviewed.

(c) Article author(s) (or their employer(s) unless otherwise stated in the text of the article) 2018. All rights reserved. No commercial use is permitted unless otherwise expressly granted.

\section{REFERENCES}

1 Köbel M, Rahimi K, Rambau PF, et al. An immunohistochemical algorithm for ovarian carcinoma typing. Int I Gynecol Pathol 2016;35:430-41

2 Kurman RJ, Carcangiu ML, Herrington CS, et al. WHO classification of tumours of female reproductive organs. Lyon: International Agency for Research on Cancer, 2014.

3 Kuhn E, Ayhan A, Shih I, et al. Ovarian Brenner tumour: a morphologic and immunohistochemical analysis suggesting an origin from fallopian tube epithelium. Eur J Cancer 2013;49:3839-49.

4 Kuhn E, Kurman RJ, Vang R, et al. TP53 mutations in serous tubal intraepithelial carcinoma and concurrent pelvic high-grade serous carcinoma - evidence supporting the clonal relationship of the two lesions.J Pathol 2012:226:421-6.

5 Kuhn E, Ayhan A, Bahadirli-Talbott A, et al. Molecular characterization of undifferentiated carcinoma associated with endometrioid carcinoma. Am J Surg Pathol 2014;38:660-5.

6 McConechy MK, Ding J, Senz J, et al. Ovarian and endometrial endometrioid carcinomas have distinct CTNNB1 and PTEN mutation profiles. Mod Pathol 2014;27:128-34.

7 Ayhan A, Mao TL, Seckin T, et al. Loss of ARID1A expression is an early molecular event in tumor progression from ovarian endometriotic cyst to clear cell and endometrioid carcinoma. Int J Gynecol Cancer 2012;22:1310-5.

8 Martins FC, Santiago I, Trinh A, et al. Combined image and genomic analysis of high-grade serous ovarian cancer reveals PTEN loss as a common driver event and prognostic classifier. Genome Biol 2014;15:526.

9 Kuhn E, Wang TL, Doberstein K, et al. CCNE1 amplification and centrosome number abnormality in serous tubal intraepithelial carcinoma: further evidence supporting its role as a precursor of ovarian high-grade serous carcinoma. Mod Pathol 2016;29:1254-61.

10 Ayhan A, Kuhn E, Wu RC, et al. CCNE1 copy-number gain and overexpression identify ovarian clear cell carcinoma with a poor prognosis. Mod Pathol 2017;30:297-303.

11 Sehdev AS, Kurman RJ, Kuhn E, et al. Serous tubal intraepithelial carcinoma upregulates markers associated with high-grade serous carcinomas including Rsf-1 (HBXAP), cyclin E and fatty acid synthase. Mod Pathol 2010;23:844-55.

12 Iwamoto M, Nakatani Y, Fugo K, et al. Napsin A is frequently expressed in clear cell carcinoma of the ovary and endometrium. Hum Pathol 2015;46:957-62.

13 Hirschowitz L, Ganesan R, McCluggage WG. WT1, p53 and hormone receptor expression in uterine serous carcinoma. Histopathology 2009;55:478-82.
14 Kommoss F, Faruqi A, Gilks CB, et al. Uterine serous carcinomas frequently metastasize to the fallopian tube and can mimic serous tubal intraepithelial carcinoma. Am J Surg Pathol 2017;41:161-70.

15 Bagby C, Ronnett BM, Yemelyanova A, et al. Clinically occult tubal and ovarian highgrade serous carcinomas presenting in uterine samples: diagnostic pitfalls and clues to improve recognition of tumor origin. Int I Gynecol Pathol 2013;32:433-43.

16 Comin CE, Saieva C, Messerini L. h-caldesmon, calretinin, estrogen receptor, and Ber-EP4: a useful combination of immunohistochemical markers for differentiating epithelioid peritoneal mesothelioma from serous papillary carcinoma of the ovary. Am I Surg Pathol 2007:31:1139-48.

17 Ordóñez NG. Value of PAX8, PAX2, claudin-4, and h-caldesmon immunostaining in distinguishing peritoneal epithelioid mesotheliomas from serous carcinomas. Mod Pathol 2013;26:553-62.

18 Chapel DB, Husain AN, Krausz T, et al. PAX8 expression in a subset of malignant peritoneal mesotheliomas and benign mesothelium has diagnostic implications in the differential diagnosis of ovarian serous carcinoma. Am J Surg Pathol 2017;41:1.

19 Laury AR, Hornick JL, Perets R, et al. PAX8 reliably distinquishes ovarian serous tumors from malignant mesothelioma. Am J Surg Pathol 2010;34:1-35.

20 Kawai T, Tominaga S, Hiroi S, et al. Peritoneal malignant mesothelioma (PMM), and primary peritoneal serous carcinoma (PPSC) and reactive mesothelial hyperplasia (RMH) of the peritoneum. Immunohistochemical and fluorescence in situ hybridisation (FISH) analyses. J Clin Pathol 2016;69:706-12.

21 Ito T, Hamasaki M, Matsumoto S, et al. p16/CDKN2A FISH in differentiation of diffuse malignant peritoneal mesothelioma from mesothelial hyperplasia and epithelial ovarian cancer. Am J Clin Pathol 2015;143:830-8.

22 Hwang HC, Sheffield BS, Rodriguez S, et al. Utility of BAP1 immunohistochemistry and 16 (CDKN2A) fish in the diagnosis of malignant mesothelioma in effusion cytology specimens. Am J Surg Pathol 2016:40:120-6.

23 Wu D, Hiroshima K, Matsumoto S, et al. Diagnostic usefulness of p16/CDKN2A FISH in distinguishing between sarcomatoid mesothelioma and fibrous pleuritis. Am J Clin Pathol 2013;139:39-46.

24 Hwang HC, Pyott S, Rodriguez S, et al. BAP1 immunohistochemistry and $p 16$ fish in the diagnosis of sarcomatous and desmoplastic mesotheliomas. Am I Surg Pathol 2016:40:714-8.

25 Moh M, Krings G, Ates D, et al. SATB2 expression distinguishes ovarian metastases of colorectal and appendiceal origin from primary ovarian tumors of mucinous or endometrioid type. Am J Surg Pathol 2016;40:419-32.

26 Strickland S, Wasserman JK, Giassi A, et al. Immunohistochemistry in the diagnosis of mucinous neoplasms involving the ovary: the added value of SATB2 and biomarker discovery through protein expression database mining. Int I Gynecol Pathol 2016;35:191-208.

27 Vang R, Gown AM, Barry TS, et al. Cytokeratins 7 and 20 in primary and secondary mucinous tumors of the ovary: analysis of coordinate immunohistochemical expression profiles and staining distribution in 179 cases. Am I Surg Pathol 2006;30:1130-9.

28 Vang R, Gown AM, Barry TS, et al. Immunohistochemistry for estrogen and progesterone receptors in the distinction of primary and metastatic mucinous tumors in the ovary: an analysis of 124 cases. Mod Pathol 2006;19:97-105.

29 De Lott LB, Morrison C, Suster S, et al. CDX2 is a useful marker of intestinal-type differentiation: a tissue microarray-based study of 629 tumors from various sites. Arch Pathol Lab Med 2005;129:1100-5.

30 Vang R, Gown AM, Wu LS, et al. Immunohistochemical expression of CDX2 in primary ovarian mucinous tumors and metastatic mucinous carcinomas involving the ovary: comparison with CK20 and correlation with coordinate expression of CK7. Mod Pathol 2006;19:1421-8.

31 Perez Montiel D, Arispe Angulo K, Cantú-de León D, et al. The value of SATB2 in the differential diagnosis of intestinal-type mucinous tumors of the ovary: primary vs metastatic. Ann Diagn Pathol 2015;19:249-52.

32 McCluggage WG. Immunohistochemistry in the distinction between primary and metastatic ovarian mucinous neoplasms. J Clin Pathol 2012;65:596-600.

33 Fadare $\mathrm{O}$, Zhao C, Khabele D, et al. Comparative analysis of Napsin A, alphamethylacyl-coenzyme A racemase (AMACR, P504S), and hepatocyte nuclear factor 1 beta as diagnostic markers of ovarian clear cell carcinoma: an immunohistochemical study of 279 ovarian tumours. Pathology 2015;47:105-11.

34 Fraggetta F, Pelosi G, Cafici A, et al. CDX2 immunoreactivity in primary and metastatic ovarian mucinous tumours. Virchows Arch 2003;443:782-6.

35 Groisman GM, Meir A, Sabo E. The value of Cdx2 immunostaining in differentiating primary ovarian carcinomas from colonic carcinomas metastatic to the ovaries. Int J Gynecol Pathol 2004;23:52-7.

36 Hu A, Li H, Zhang L, et al. Differentiating primary and extragenital metastatic mucinous ovarian tumours: an algorithm combining PAX8 with tumour size and laterality. J Clin Pathol 2015;68:522-8.

37 Karnezis AN, Wang Y, Ramos P, et al. Dual loss of the SWI/SNF complex ATPases SMARCA4/BRG1 and SMARCA2/BRM is highly sensitive and specific for small cell carcinoma of the ovary, hypercalcaemic type. J Pathol 2016;238:389-400.

38 Ramalingam P, Malpica A, Silva EG, et al. The use of cytokeratin 7 and EMA in differentiating ovarian yolk sac tumors from endometrioid and clear cell carcinomas. Am J Surg Pathol 2004;28:1499-505. 
39 Cao D, Guo S, Allan RW, et al. SALL4 is a novel sensitive and specific marker of ovarian primitive germ cell tumors and is particularly useful in distinguishing yolk sac tumor from clear cell carcinoma. Am J Surg Pathol 2009;33:894-904.

40 Ravishankar S, Malpica A, Ramalingam P, et al. Yolk sac tumor in extragonadal pelvic sites: still a diagnostic challenge. Am J Surg Pathol 2017;41:1-11.

41 Kuhn E, Kurman RJ, Sehdev AS, et al. Ki-67 labeling index as an adjunct in the diagnosis of serous tubal intraepithelial carcinoma. Int J Gynecol Pathol 2012;31:416-22

42 Vang R, Visvanathan K, Gross A, et al. Validation of an algorithm for the diagnosis of serous tubal intraepithelial carcinoma. Int J Gynecol Pathol 2012;31:243-53.

43 Kuhn E, Wu RC, Guan B, et al. Identification of molecular pathway aberrations in uterine serous carcinoma by genome-wide analyses. J Nat/ Cancer Inst 2012;104:1503-13.

44 Chiang S, Soslow RA. Updates in diagnostic immunohistochemistry in endometrial carcinoma. Semin Diagn Pathol 2014:31:205-15.

45 Mao TL, Ayhan A, Kuo KT, et al. Immunohistochemical study of endometrial high-grade endometrioid carcinoma with or without a concurrent low-grade component: implications for pathogenetic and survival differences. Histopathology 2015:67:474-82

46 Lim D, Ip PP, Cheung AN, et al. Immunohistochemical comparison of ovarian and uterine endometrioid carcinoma, endometrioid carcinoma with clear cell change, and clear cell carcinoma. Am J Surg Pathol 2015;39:1061-9.

47 Tafe LJ, Garg K, Chew I, et al. Endometrial and ovarian carcinomas with undifferentiated components: clinically aggressive and frequently underrecognized neoplasms. Mod Pathol 2010;23:781-9.

48 Shah VI, Ramalingam P, McCluggage WG. CD34 expression in undifferentiated endometrial carcinoma. Histopathology 2016;69:894-7.

49 Ramalingam P, Croce S, McCluggage WG. Loss of expression of SMARCA4 (BRG1), SMARCA2 (BRM) and SMARCB1 (INI1) in undifferentiated carcinoma of the endometrium is not uncommon and is not always associated with rhabdoid morphology. Histopathology 2017;70:359-66.

50 Tipps AM, Plaxe SC, Weidner N. Endometrioid carcinoma with a low-grade spindle cell component: a tumor resembling an adnexal tumor of probable Wolffian origin. Ann Diagn Pathol 2011;15:376-81.

51 Tornos C, Silva EG, Ordonez NG, et al. Endometrioid carcinoma of the ovary with a prominent spindle-cell component, a source of diagnostic confusion. A report of 14 cases. Am J Surg Pathol 1995;19:1343-53.

52 Esteller M, Catasus L, Matias-Guiu X, et al. hMLH1 promoter hypermethylation is an early event in human endometrial tumorigenesis. Am J Pathol 1999;155:1767-72.

53 Norimatsu Y, Moriya T, Kobayashi TK, et al. Immunohistochemical expression of PTEN and beta-catenin for endometrial intraepithelial neoplasia in Japanese women. Ann Diagn Pathol 2007;11:103-8.

54 Joiner AK, Quick CM, Jeffus SK. Pax2 expression in simultaneously diagnosed WHO and EIN classification systems. Int J Gynecol Pathol 2015;34:40-6.

55 Allison $\mathrm{KH}$, Upson K, Reed SD, et al. PAX2 loss by immunohistochemistry occurs early and often in endometrial hyperplasia. Int J Gynecol Pathol 2012;31:159-67.

56 Monte NM, Webster KA, Neuberg D, et al. Joint loss of PAX2 and PTEN expression in endometrial precancers and cancer. Cancer Res 2010;70:6225-32.

57 Quick CM, Laury AR, Monte NM, et al. Utility of PAX2 as a marker for diagnosis of endometrial intraepithelial neoplasia. Am J Clin Pathol 2012;138:678-84.

58 Usubutun A, Mutter GL, Saglam A, et al. Reproducibility of endometrial intraepithelial neoplasia diagnosis is good, but influenced by the diagnostic style of pathologists. Mod Pathol 2012;25:877-84.

59 Ayhan A, Mao TL, Suryo Rahmanto Y, et al. Increased proliferation in atypical hyperplasia/endometrioid intraepithelial neoplasia of the endometrium with concurrent inactivation of ARID1A and PTEN tumour suppressors.J Pathol Clin Res 2015;1:186-93.

60 Mutter GL, Monte NM, Neuberg D, et al. Emergence, involution, and progression to carcinoma of mutant clones in normal endometrial tissues. Cancer Res 2014;74:2796-802

61 Aggarwal N, Bhargava R, Elishaev E. Uterine adenosarcomas: diagnostic use of the proliferation marker Ki-67 as an adjunct to morphologic diagnosis. Int J Gynecol Pathol 2012;31:447-52.

62 Koontz JI, Soreng AL, Nucci M, et al. Frequent fusion of the JAZF1 and JJAZ1 genes in endometrial stromal tumors. Proc Natl Acad Sci U S A 2001;98:6348-53.

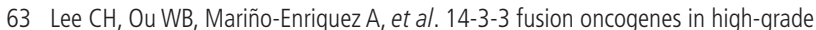
endometrial stromal sarcoma. Proc Natl Acad Sci U S A 2012;109:929-34.

64 Suzuki S, Tanioka F, Minato H, et al. Breakages at YWHAE, FAM22A, and FAM22B loci in uterine angiosarcoma: a case report with immunohistochemical and genetic analysis. Pathol Res Pract 2014;210:130-4.

65 Kurihara S, Oda Y, Ohishi Y, et al. Endometrial stromal sarcomas and related highgrade sarcomas: immunohistochemical and molecular genetic study of 31 cases. Am J Surg Pathol 2008;32:1228-38.

66 Lee CH, Ali RH, Rouzbahman M, et al. Cyclin D1 as a diagnostic immunomarker for endometrial stromal sarcoma with YWHAE-FAM22 rearrangement. Am J Surg Pathol 2012;36:1562-70.

67 Lee $\mathrm{CH}$, Hoang LN, Yip S, et al. Frequent expression of KIT in endometrial stromal sarcoma with YWHAE genetic rearrangement. Mod Pathol 2014;27:751-7.
68 Halbwedl I, Ullmann R, Kremser ML, et al. Chromosomal alterations in low-grade endometrial stromal sarcoma and undifferentiated endometrial sarcoma as detected by comparative genomic hybridization. Gynecol Oncol 2005;97:582-7.

69 Oliva E. Cellular mesenchymal tumors of the uterus: a review emphasizing recent observations. Int J Gynecol Pathol 2014;33:374-84.

70 Ali RH, Rouzbahman M. Endometrial stromal tumours revisited: an update based on the 2014 WHO classification. J Clin Pathol 2015;68:325-32.

71 Zhu XQ, Shi YF, Cheng XD, et al. Immunohistochemical markers in differential diagnosis of endometrial stromal sarcoma and cellular leiomyoma. Gynecol Oncol 2004;92:71-9.

72 Chesnais AL, Watkin E, Beurton D, et al. [Myxoid mesenchymal tumors of uterus: endometrial stromal and smooth muscle tumors, myxoid variant]. Ann Pathol 2011;31:152-8

73 Oliva E, Young RH, Amin MB, et al. An immunohistochemical analysis of endometrial stromal and smooth muscle tumors of the uterus: a study of 54 cases emphasizing the importance of using a panel because of overlap in immunoreactivity for individual antibodies. Am J Surg Pathol 2002:26:403-12.

74 O'Neill CJ, McBride HA, Connolly LE, et al. Uterine leiomyosarcomas are characterized by high p16, p53 and MIB1 expression in comparison with usual leiomyomas, leiomyoma variants and smooth muscle tumours of uncertain malignant potential. Histopathology 2007;50:851-8.

75 Chen L, Yang B. Immunohistochemical analysis of p16, p53, and Ki-67 expression in uterine smooth muscle tumors. Int J Gynecol Pathol 2008;27:326-32.

76 Mittal K, Demopoulos RI. MIB-1 (Ki-67), p53, estrogen receptor, and progesterone receptor expression in uterine smooth muscle tumors. Hum Pathol 2001:32:984-7.

77 Darragh TM, Colgan TJ, Cox JT, et al. The lower anogenital squamous terminology standardization project for HPV-associated lesions: background and consensus recommendations from the College of American Pathologists and the American Society for Colposcopy and Cervical Pathology. Arch Pathol Lab Med 2012;136:1266-97.

78 Maniar KP, Sanchez B, Paintal A, et al. Role of the biomarker p16 in Downgrading -IN 2 diagnoses and predicting higher-grade lesions. Am J Surg Pathol 2015;39:1708-18.

79 Guedes AC, Brenna SM, Coelho SA, et al. p16(INK4a) Expression does not predict the outcome of cervical intraepithelial neoplasia grade 2. Int J Gynecol Cancer 2007:17:1099-103.

80 Miralpeix E, Genovés J, Maria Solé-Sedeño J, et al. Usefulness of p16(INK4a) staining for managing histological high-grade squamous intraepithelial cervical lesions. Mod Pathol 2017:30:304-10

81 Yang EJ, Quick MC, Hanamornroongruang S, et al. Microanatomy of the cervical and anorectal squamocolumnar junctions: a proposed model for anatomical differences in HPV-related cancer risk. Mod Pathol 2015:28:994-1000.

82 Herfs M, Parra-Herran C, Howitt BE, et al. Cervical squamocolumnar junctionspecific markers define distinct, clinically relevant subsets of low-grade squamous intraepithelial lesions. Am J Surg Pathol 2013;37:1311-8.

83 Mills AM, Paquette C, Terzic T, et al. CK7 Immunohistochemistry as a predictor of CIN1 progression: a retrospective study of patients from the quadrivalent HPV vaccine trials. Am J Surg Pathol 2017;41:143-52.

84 Paquette C, Mills AM, Stoler MH. Predictive value of cytokeratin 7 immunohistochemistry in cervical low-grade squamous intraepithelial lesion as a marker for risk of progression to a high-grade lesion. Am J Surg Pathol 2016:40:236-43.

85 Huang EC, Tomic MM, Hanamornroongruang S, et al. p16ink4 and cytokeratin 7 immunostaining in predicting HSIL outcome for low-grade squamous intraepithelial lesions: a case series, literature review and commentary. Mod Pathol 2016;29:1501-10.

86 Loureiro J, Oliva E. The spectrum of cervical glandular neoplasia and issues in differential diagnosis. Arch Pathol Lab Med

2014;138:453-83.

87 McCluggage WG. New developments in endocervical glandular lesions. Histopathology 2013;62:138-60.

88 Young RH, Clement PB. Endocervical adenocarcinoma and its variants: their morphology and differential diagnosis. Histopathology 2002;41:185-207.

89 McCluggage WG, Sumathi VP, McBride HA, et al. A panel of immunohistochemical stains, including carcinoembryonic antigen, vimentin, and estrogen receptor, aids the distinction between primary endometrial and endocervical adenocarcinomas. Int J Gynecol Pathol 2002;21:11-15.

90 Carleton C, Hoang L, Sah S, et al. A detailed immunohistochemical analysis of a large series of cervical and vaginal gastric-type adenocarcinomas. Am J Surg Pathol 2016:40:636-44.

91 Park KJ, Kiyokawa T, Soslow RA, et al. Unusual endocervical adenocarcinomas: an immunohistochemical analysis with molecular detection of human papillomavirus. Am J Surg Pathol 2011;35:633-46.

92 Kenny SL, McBride HA, Jamison J, et al. Mesonephric adenocarcinomas of the uterine cervix and corpus: HPV-negative neoplasms that are commonly PAX8, CA125, and HMGA2 positive and that may be immunoreactive with TTF1 and hepatocyte nuclear factor 1- $\beta$. Am J Surg Pathol 2012;36:799-807. 
93 Cortes-Ciriano I, Lee S, Park WY, et al. A molecular portrait of microsatellite instability across multiple cancers. Nat Commun 2017:8:15180.

94 Broaddus RR, Lynch HT, Chen LM, et al. Pathologic features of endometrial carcinoma associated with HNPCC: a comparison with sporadic endometrial carcinoma. Cancer 2006;106:87-94.

95 Carcangiu ML, Radice $\mathrm{P}$, Casalini $\mathrm{P}$, et al. Lynch syndrome-related endometrial carcinomas show a high frequency of nonendometrioid types and of high FIGO grade endometrioid types. Int J Surg Pathol 2010;18:21-6.

96 Garg K, Leitao MM, Kauff ND, et al. Selection of endometrial carcinomas for DNA mismatch repair protein immunohistochemistry using patient age and tumor morphology enhances detection of mismatch repair abnormalities. Am J Surg Pathol 2009:33:925-33.

97 McConechy MK, Talhouk A, Li-Chang HH, et al. Detection of DNA mismatch repair (MMR) deficiencies by immunohistochemistry can effectively diagnose the microsatellite instability (MSI) phenotype in endometrial carcinomas. Gynecol Oncol 2015;137:306-10.

98 Ryan P, Mulligan AM, Aronson M, et al. Comparison of clinical schemas and morphologic features in predicting Lynch syndrome in mutation-positive patients with endometrial cancer encountered in the context of familial gastrointestinal cancer registries. Cancer 2012;118:681-8.

$99 \mathrm{Lu} \mathrm{KH}$, Schorge JO, Rodabaugh KJ, et al. Prospective determination of prevalence of lynch syndrome in young women with endometrial cancer. J Clin Oncol 2007;25:5158-64.

100 Zhang L. Immunohistochemistry versus microsatellite instability testing for screening colorectal cancer patients at risk for hereditary nonpolyposis colorectal cancer syndrome. Part II. The utility of microsatellite instability testing. J Mol Diagn 2008;10:301-7.

101 Wang Y, Shi C, Eisenberg R, et al. Differences in microsatellite instability profiles between endometrioid and colorectal cancers: a potential cause for false-negative results? J Mol Diagn 2017;19:57-64.

102 Segev Y, lqbal J, Lubinski J, et al. The incidence of endometrial cancer in women with BRCA1 and BRCA2 mutations: an international prospective cohort study. Gynecol Oncol 2013;130:127-31.

103 Soslow RA, Han G, Park KJ, et al. Morphologic patterns associated with BRCA1 and BRCA2 genotype in ovarian carcinoma. Mod Pathol 2012;25:625-36.

104 Hussein YR, Ducie JA, Arnold AG, et al. Invasion patterns of metastatic extrauterine high-grade serous carcinoma with BRCA germline mutation and correlation with clinical outcomes. Am J Surg Pathol 2016;40:404-9.

105 Meisel JL, Hyman DM, Garg K, et al. The performance of BRCA immunohistochemistry for detecting germline, somatic, and epigenetic BRCA1 loss in high-grade serous ovarian cancer. Ann Oncol 2014;25:2372-8.

106 Endris V, Stenzinger A, Pfarr N, et al. NGS-based BRCA1/2 mutation testing of highgrade serous ovarian cancer tissue: results and conclusions of the first international round robin trial. Virchows Arch 2016:468:697-705.

107 Konecny GE. Understanding exceptional responses to poly (ADP-ribose) polymerase inhibition in sporadic ovarian cancer. J Clin Oncol 2017:35:1151-3.

108 Fong PC, Yap TA, Boss DS, et al. Poly(ADP)-ribose polymerase inhibition: frequent durable responses in BRCA carrier ovarian cancer correlating with platinum-free interval. J Clin Oncol 2010;28:2512-9.

109 Byrski T, Gronwald J, Huzarski T, et al. Pathologic complete response rates in young women with BRCA1-positive breast cancers after neoadjuvant chemotherapy. J Clin Oncol 2010;28:375-9.

110 Le DT, Durham JN, Smith KN, et al. Mismatch repair deficiency predicts response of solid tumors to PD-1 blockade. Science 2017;357:409-13.

111 Tornillo L, Moch H, Diener PA, et al. CDX-2 immunostaining in primary and secondary ovarian carcinomas. J Clin Pathol 2004;57:641-3.
112 Tabrizi AD, Kalloger SE, Köbel M, et al. Primary ovarian mucinous carcinoma of intestinal type: significance of pattern of invasion and immunohistochemical expression profile in a series of 31 cases. Int J Gynecol Pathol 2010;29:99-107.

113 Saad RS, Ismiil N, Dubé V, et al. CDX-2 expression is a common event in primary intestinal-type endocervical adenocarcinoma. Am J Clin Pathol 2009:132:531-8.

114 Wani Y, Notohara K, Saegusa M, et al. Aberrant Cdx2 expression in endometrial lesions with squamous differentiation: important role of $\mathrm{Cdx} 2$ in squamous morula formation. Hum Pathol 2008;39:1072-9.

115 Houghton O, Connolly LE, McCluggage WG. Morules in endometrioid proliferations of the uterus and ovary consistently express the intestinal transcription factor CDX2. Histopathology 2008;53:156-65.

116 Ordóñez NG. The diagnostic utility of immunohistochemistry and electron microscopy in distinguishing between peritoneal mesotheliomas and serous carcinomas: a comparative study. Mod Pathol 2006:19:34-48.

117 Oe S, Hasegawa K, Nagase S, et al. Expression of podoplanin in epithelial ovarian carcinomas and its potential as a marker for clear cell adenocarcinoma. Int I Gynecol Pathol 2010;29:405-10

118 Chu AY, Litzky LA, Pasha TL, et al. Utility of D2-40, a novel mesothelial marker, in the diagnosis of malignant mesothelioma. Mod Pathol 2005;18:105-10.

119 Chang MC, Vargas SO, Hornick JL, et al. Embryonic stem cell transcription factors and D2-40 (podoplanin) as diagnostic immunohistochemical markers in ovarian germ cell tumors. Int J Gynecol Pathol 2009;28:347-55.

120 Clark BZ, Beriwal S, Dabbs DJ, et al. Semiquantitative GATA-3 immunoreactivity in breast, bladder, gynecologic tract, and other cytokeratin 7-positive carcinomas. Am J Clin Pathol 2014;142:64-71.

121 Engelsen IB, Stefansson IM, Akslen LA, et al. GATA3 expression in estrogen receptor alpha-negative endometrial carcinomas identifies aggressive tumors with high proliferation and poor patient survival. Am J Obstet Gynecol 2008;199:543.e1-e7.

122 Esheba GE, Longacre TA, Atkins KA, et al. Expression of the urothelial differentiation markers GATA3 and placental S100 (S100P) in female genital tract transitional cel proliferations. Am J Surg Pathol 2009;33:347-53.

123 Chang A, Amin A, Gabrielson E, et al. Utility of GATA3 immunohistochemistry in differentiating urothelial carcinoma from prostate adenocarcinoma and squamous cell carcinomas of the uterine cervix, anus, and lung. Am J Surg Pathol 2012;36:1472-6.

124 Roma AA, Goyal A, Yang B. Differential expression patterns of GATA3 in uterine mesonephric and nonmesonephric lesions. Int J Gynecol Pathol 2015;34:480-6.

125 Howitt BE, Emori MM, Drapkin R, et al. GATA3 is a sensitive and specific Marker of benign and malignant mesonephric lesions in the lower Female genital tract. Am J Surg Pathol 2015;39:1411-9.

126 Ervine A, Leung S, Gilks CB, et al. Thyroid transcription factor-1 (TTF-1) immunoreactivity is an adverse prognostic factor in endometrioid adenocarcinoma of the uterine corpus. Histopathology 2014;64:840-6.

127 Siami K, McCluggage WG, Ordonez NG, et al. Thyroid transcription factor-1 expression in endometrial and endocervical adenocarcinomas. Am J Surg Pathol 2007:31:1759-63.

128 Graham AD, Williams AR, Salter DM. TTF-1 expression in primary ovarian epithelial neoplasia. Histopathology 2006:48:764-5.

129 Kubba LA, McCluggage WG, Liu J, et al. Thyroid transcription factor-1 expression in ovarian epithelial neoplasms. Mod Pathol 2008;21:485-90.

130 Zhang PJ, Gao HG, Pasha TL, et al. TTF-1 expression in ovarian and uterine epithelial neoplasia and its potential significance, an immunohistochemical assessment with multiple monoclonal antibodies and different secondary detection systems. Int J Gynecol Pathol 2009;28:10-18. 